the uvula, so that both were described under the term of lobus uvulae (Cunningham) -is without justification, as the tonsil is morphologically the anterior part of the alae lobuli medii, or the hemispheres. Elliot Smith has also pointed out the fallacy of dividing the suprapyramidal part of the vermis into a clivus, folium cacuminis and tuber valvulae, all these constituting one common structure. The extent to which the present terminology is misleading is shown by the confusion which resulted when experimental physiologists cndeavored to apply the results of their studies on lower animals to the cerebellum of man. We thus find, for example, a man of such eminence as Dr. Rothmann of Berlin identifying the lobulus paramedianus (the tonsil or amygdalus in man) with the gyrus semilunaris inferior; the crus secundum (the lobulus biventer) with the gyrus semilunaris superior; the formatio vermicularis (the lobulus flocculi) with the lobulus biventer; and the crus primum (the lobulus semilunaris superior) with the lobus quadrangularis.

Dr. William Alexander Jones, Minneapolis: Dr. Fuller's cfforts to simplify the nomenclature and terminology on the filser system of the brain should meet with general approval. For many years I taught from some of Dr. Fuller's models and I still study his architecture of the brain with confidence. The illustrations are very clear and they make the brain structure easier to remember.

DR. WALter B. SwIFT, Boston: I have taught anatomy of the brain and psychoneuropathology for four years. When I went into this course there were charts and vague models and vague representations of tissue. I soon dispensed with all that, and supplied real brain and tissue sections for study. This has made the teaching much more simple and gives the student a chance to have in mind the visual pictures of the real tissue just as it is, not merely visual pictures of charts and models. Dr. Fuller has taken this matter one step farther; and shown not only the tissue and sections, but the relations of correlated tracts, and made that subject much easier to learn than from mere flat cross section. I shall use this method more than ever next year.

Dr. D. Booth, St. Louis: The use of proper names in connection with parts of the anatomy is very poor policy, because in that case the student has that abstract fact to remember instead of having the data formulated in his mind. I have always thought it easier to say iter a tertio ad quartum ventriculum, than to say aqueduct of Sylvius, because we are dealing with the things which we are trying to demonstrate. Association is a great aid to memory.

\section{PERFORATED ULCERS OF THE STOMACH AND DUODENUM *}

\section{RAYMOND P. SULLIVAN, M.D. BROOKLYN}

Perforation of a gastric or duodenal ulcer is a most serious condition. The prognosis is extremely grave unless recognized early and quickly followed by direct and heroic treatment. Of all the acute maladies to which humanity is heir, none is more grave and none more spectacular when attended by successful treatment. The treatment is purely surgical. However, Moynihan ${ }^{1}$ reports two cases successfully treated by medical means during the acute stage; but in each instance the cases came to operation at a later date. We occasionally hear of deaths from "acute indigestion," but proper investigation would prove, in the majority of instances, that there was a perforation of either a gastric or duodenal ulcer. This should not occur at the present time. The burden of diagnosis will rest with the general practitioner and, even though an accurate diagnosis may not be made, at least there

* Read before the Section, on Surgery, General. and Abdominal, at the Sixty.Seventh Annual Session of the American Medical Association, Detroit, June, 1916.

1. Moynihan: Abdominal Operations, i, 165. are always sufficiently grave signs to demand a surgical consultation and intervention.

The etiology of perforation is that of ulcer in general plus its acute termination. It results from a local necrosis and may be due to trophic disturbances or bacterial invasions, or it may follow trauma. However, a previous chronic ulcer is usually present. The immediate cause of the perforation may result from one of many factors. In all series reported, muscular exertion, trauma, food or alcohol ingestion are given as the immediate factors conducive to perforation. The series here presented will confirm this. That an infective process predominates seems to be shown by the fact that so frequently there is a two-cycle phenomena of pain. A premonitory pain of greater or lesser degree was present in fourteen instances in this series. I believe this is due to a rapid necrosis, causing peritoneal irritation and spasm and hence the pain. Just how long the peritoneum will hold against this process cannot be determined exactly. The "big pain" has followed on an average of eight hours in each instance.

The chief symptoms which lead to a correct diagnosis in these cases are: (1) pain, (2) tenderness, (3) vomiting, (4) rigidity, (5) history of previous ulcer symptoms, (6) anxiety and restleșsness, and (7) increasing pulse and rise of temperature. This enumeration is considered in the order of frequency observed in the series here reported.

Pain is sudden and violent. Its character is variously described by patients as "sharp," "cutting," "burning," or "bursting and stabbing." The prominent characteristic is the fact that it is unyielding. Its localization is mostly epigastric and supra-umbilical. Its quick radiation at times to the right iliac fossa has frequently led physicians and surgeons to consider an acute appendicitis; but careful inquiry of its early appearance most frequently localized it in the epigastric and right hypochondriac regions. Its radiation may be in any direction. The prominent factor is that the pain is excruciating and increasing in severity to such a degree that the patient is unwilling to submit to much examination.

Tenderness is unmistakable. It is acute and easily elicited. When the case is seen early, a careful and gentle palpation will lead to a marked tenderness over the site of the perforation. The site of maximum tenderness in this series varied mostly in the gastric perforations. There were fifteen duodenal perforations, and in twelve of them the most tender area was in the right hypochondriac region. In three, the right iliac fossa was made out as the most tender area; hence the improper clue to a correct diagnosis. Of the five gastric cases the maximum tenderness was all epigastric, and in one of these it was to the left of the median line.

Vomiting occurs very early, either reflex or induced, and is a valuable symptom. Frequently it gives slight relief to the agony at first, but later only increases the pain. However, I cannot say that vomiting will lead to any positive sign; for it may be present in almost any abdominal condition. Eliot ${ }^{2}$ reports two cases in which there was no vomiting at all. In this series of cases, both gastric and duodenal, vomiting occurred very early and in every case. The character of the vomitus varied with the amount of food previously taken and also with the site of the ulcer. In duodenal

2. Eliot: Ann. Surg., lv, 594. 
cases the vomitus was greater in amount than in the gastric cases.

The second symptom of greatest value is that of rigidity of the abdominal muscles. Its appearance is early and its increase always in proportion to the length of time following the onset of symptoms. Deaver" very aptly says, "There is no condition in which the upper abdominal rigidity is so early and so marked as in perforated ulcer." In our experience with these cases we have relied chiefly on the character of the pain and the amount of rigidity as the principal indications of perforation. The "boardlike" type is the most significant guide, and the fact that it is continuous points to a progressive peritonitis and hence is of great value as a diagnostic sign.

When a patient is seen suffering from so acute abdominal pain and has this characteristic rigidity, the history of previous indigestion of ulcer type is usually obtainable. In this series of cases only one instance is noted in which previous ulcer symptoms failed to be elicited.

In this case there was a history of trauma eight days before perforation and the patient was confined to bed, being treated for "contusion of the abdomen," following an injury. On the morning of the eighth day after the injury, he was informed by his physician that he could go about, but was warned against any exertion or violent exercise. The patient said he felt so good that he determined to return to work and did so. During the afternoon, while lifting a "heavy case onto a wagon" he was taken with the terrible pain in the pit of the stomach, which increased every minute and necessitated calling an ambulance. $\mathrm{He}$ was taken to the hospital and we operated three hours later, finding a perforated gastric ulcer on the anterior wall about $4 \frac{1}{2}$ inches from the pylorus (Case 12).

In all other cases the previous ulcer symptoms were present. In eleven a diagnosis of chronic ulcer had been made and all had been treated by medical means with the uniform result of temporary relief for a variable length of time. This would seem to indicate that surgical treatment should be instituted for chronic ulcer, if for nothing more than a prophylaxis against perforation.

In cases of so grave a nature one might expect that shock and its associated signs would be manifest. However, this was not found in this series. The patients, on the contrary, seemed to be in fairly good general physical condition. In only one instance, and that one was seen late, was there any evidence of shock. Yet these patients all presented an appearance of anxiety and restlessness which signified that some great calamity had taken place within them. Their attitude was one of intense suffering. But the average pulse rate in this series was 90 . The average temperature was $99.8 \mathrm{~F}$. The average respiration was 34 . I consider the increased rate of respiration an objective sign of value, because of the attempt on the part of Nature to restrain the movements of the diaphragm, and thus lessen the pain. However, if shock is present, it is not a contraindication to immediate operation. If the patient presents signs of perforation, immediate surgical intervention is indicated, and every hour of delay decreases the chances of recovery to a marked degree. In Case 10 of this series the man arrived at the hospital fourteen hours after the onset of the intense pain. He was in profound shock, and exploration revealed a large perforated gastric ulcer with a general perito-

3. Deaver, J. B.: Posterior Gastrojejunostomy in Acute Perforative Uicer of the Stomach and Duodenum, Tнв Journal A. M. A., July 12, 1913 , p. 75 . nitis. He received very little anesthetic and the perforation was closed. His reaction was good. He lived five days. A reperforation superimposed on his general peritonitis was the cause of his death.

It is a noteworthy fact that in this series not once is "absence of liver dulness" recorded. It is therefore considered that this sign is not as valuable as we might expect. However, in a series of cases recently reported," "modification of liver dulness" was noted in 127 out of 175 cases of perforated gastric ulcer. The amount of distention which might cause the modification of liver dulness has frequently been attributed to the escape of gas into the peritoneal cavity. This will be true if the amount of gas is sufficient. However, it is my opinion that when there is an absence or modification of liver dulness the cause is chiefly that of a progressed peritonitis. Hence, if this condition can be made out, its value is simply that of an unfavorable prognostic sign. Numerous symptoms and evidences other than those mentioned may be found, but the time spent in eliciting them will only delay operation and hence increase the chances for a fatal outcome.

Given a patient who is taken suddenly ill with an acute cutting or piercing epigastric pain which is continuously agonizing and accompanied by a unique or marked rigidity of the abdominal muscles, particularly of the upper half, and from whom a previous history of indigestion is obtained, the most imperative indication is operation. Differential diagnosis may have scientific value, but the moral value is dependent on early operation. There is no excuse for delay in the presence of such symptoms. It is true that acute appendicitis, perforation of the gallbladder, acute pancreatitis and acute intestinal obstruction may give an almost identical picture. However, the treatment in each of these conditions will be early operation. It is, therefore, necessary that a surgeon should see these cases as soon as possible.

Between September, 1910, and March, 1916, it was my opportunity to operate in twenty cases of perforated ulcer. In this series there were fifteen duodenal and five gastric perforations. Death resulted in one case of perforated gastric ulcer. There were six females and fourteen males. The average age was 34 years. The average duration of acute symptoms before operation was about six hours. The treatment in each case was surgical.

In the gastric cases the site of the perforation was found to be in one instance (Case 2) on the lesser curvature and the treatment used was excision and suture closure.

In another case the perforation was on the posterior wall . (Case 11) about $1 \frac{1}{2}$ inches from the pylorus. The attempt to close this, both by an approach through the lesser sac and by a transgastric method, failed and finally we did a pylorectomy and posterior gastro-enterostomy. This patient had been under observation and a diagnosis of chronic gastric ulcer was made. He was ordered to be prepared for operation, Nov. 29, 1913. At 8 p. m., November 28 , he received a gastric lavage. At 4 a. m., on the $29 \mathrm{th}$, he was taken with severe epigastric pain soon followed by vomiting which contained a good deal of blood-streaked mucus. At $6: 30 \mathrm{a} . \mathrm{m}$., a second and more intense epigastric pain came on suddenly and continucd with increasing severity. I saw him at $7: 30$ a. m., and the boardiike rigidity of the abdomen was present with extreme tenderness in the epigastric region. He was quickly submitted to immediate operation. When the peritoneum was opened, a thin serous fluid escaped, but no blood clot or mucus. The lesser sac was distended and immedi-

4. Edinburgh Med. Jour., December, 1914, p. 461. 
ately opened. A large blood clot was removed, followed by mucus, blood and bile. The perforation was found to be about $1 / 16$ inch wide and plugged with a clot of blood. Our attempt to close it was unsuccessful, and in view of his good general condition and also from the fact that the general peritoneal cavity was not soiled by food particles, we proceeded with the pylorectomy and suture posterior no loop gastro-enterostomy. Drainage was instituted and the wound closed from below upward to the drain. His convalescence was stormy for two days, but soon returned to normal. His condition today is excellent. He has gained 26 pounds and enjoys his food without any distress.

In three cases $(7,10$ and 12) the perforations were on the anterior wall. In Case 7 , the site of the perforation was in the pars media and close to the greater curve or about 3 inches from the pylorus. In this case the history of acute pain was five hours in duration, and the vomitus contained a good deal of blood. When the peritoneum was opened there was an escape of gas and many blood clots, with a large amount of mucoid material and a few food particles. The perforation was small and closed by a purse string suture of chromic catgut with reinforcement by three Lembert sutures of fine linen. This was followed by a thorough cleansing of the abdominal cavity by means of the Pool- a patient across the ward. He was arrested in his attempt by the nurse. This displeased him very much and brought forth a vigorous complaint when seen on morning rounds. But he was not to go hungry. Somehow he managed to get word to his wife and she smuggled a piece of beefsteak to him that afternoon. Fearing detection, he must have masticated his meat very poorly and had finished about two thirds of it before he was discovered by the nurse. We did nothing when this was reported. However, about an hour and a half later he was seized with intense pain and vomiting. When his abdominal wound was examined by $\mathrm{Dr}$. Coakley, the house surgeon, it was found to have been ruptured and that there was an escape of gastric contents. We reopened the entire wound and found that a reperforation had occurred. Some meat was found in the stomach and also particles in the abdomen. A rubber tube was inserted into the perforation and a gauze pack placed around its base, hoping for a gastric fistula. The patient died fourteen hours later of a general peritonitis.

In Case 12, the perforation was situated on the anterior wall about the junction of the pars cardia and the pars media. This case resulted from trauma and the perforation occurred eight hours after the injury. The immediate cause of the perforation was attributed to muscular exertion.

PERFORATION OF GASTRIC AND DUODENAL ULCERS

\begin{tabular}{|c|c|c|c|c|c|c|c|c|c|c|}
\hline $\begin{array}{c}\text { Case } \\
\text { No. }\end{array}$ & Sex & Age & $\begin{array}{l}\text { Hours of } \\
\text { Symptoms }\end{array}$ & $\begin{array}{c}\text { Preoperative } \\
\text { Diagnosis }\end{array}$ & $\begin{array}{c}\text { Onerative } \\
\text { Findings }\end{array}$ & Operation & $\begin{array}{c}\text { Drain } \\
\text { age }\end{array}$ & $\begin{array}{l}\text { Soft Diet } \\
\text { After Op. }\end{array}$ & $\begin{array}{l}\text { Dis. from } \\
\text { Hospital }\end{array}$ & Result \\
\hline 1 & M. & 35 & 6 & Pf. d. d. ul... & Pf. d. al, $u l . . .$. & Perforation closed....... & Yes & 9 days & 22 days & Gained weight: no symptoms \\
\hline 2 & F. & 51 & 10 & Pf. gast. ul.. & Pf. gast. ul.. & Fxcision and closure.... & Yes & 10 days & 30 days & No disturb.; good health \\
\hline 3 & M. & 23 & 8 & Acute append. & Pf. d. d. ul.. & Closure and Gas.-Ent.... & Yes & 5 days & 15 days & 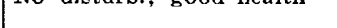 \\
\hline 4 & M. & 32 & 6 & Pf. d. d. ul...... & Pf. d. d. vl.... & Perforation closed....... & Yes & 8 days & 21 days & Condition perfect \\
\hline 5 & M. & 22 & 4 & Acute append.... & Pf. d. d. ul. & Perforation closed....... & Yes & 8 days & 24 days & \\
\hline 6 & F. & 41 & 4 & Pf. gast. ul....... & Pf. d. d. ul. & Closure and Gas.-Fnt.... & Yes & 6 days & 26 days & \\
\hline 7 & F. & 19 & 5 & Pf. d. d. ul...... & Pf. gast. vl. & Perforation closed....... & Yes & 10 days & 22 days & \\
\hline 8 & M. & 30 & 6 & Pf. gast. ul...... & Pf. d. d. ul. & Closure and Gas.-Fnt... & Yes & 6 days & 20 days & \\
\hline 0 & F. & 26 & 5 & Acute append.... & Pf. d. d. ul.. & Closure and Gas.-Fnt.... & No & 5 days & 16 days & \\
\hline 10 & M. & 56 & 14 & Pf. gast. ul...... & Pf. gast. ul.... & Perforation closed........ & Yes & & & Died \\
\hline 11 & M. & 50 & 8 & Pf. gast. ul...... & Pf. gast. ul.... & $\begin{array}{l}\text { Pylorectomy and Gas.- } \\
\text { Fint. }\end{array}$ & Yes & 10 days & 28 days & Gained weight; good health \\
\hline 12 & M. & 25 & 4 & Pf. d. d. ul. . & Pf. gast. ul.. & Perforation closed...... & No & 7 days & 14 days & Some distress \\
\hline 13 & M. & 38 & 6 & Pf. d. d. ul. & Pf. d. d. ul. & Closure and Gas.-Ent... & Y̌es & 6 days & 24 days & Gained in weight; excellent \\
\hline 14 & M. & 33 & 4 & Pf. gast. ul...... & Pf. d. d, ul. & Closure and Gas.-Ent.... & No & 5 days & 21 days & Little gastric distress after \\
\hline 15 & F. & 38 & 7 & Pf. d. d. ul... & Pf. d. d. ul.. & Closure and Gas.-Ent... & Yes & 5 days & 23 days & Gained in weight; excellent \\
\hline 16 & M. & 24 & $51 / 2$ & Pf. d. d. vil... & Pf. d. d. ul. & Perforation closed... & No & 8 days & 18 days & $\begin{array}{l}\text { nealth } \\
\text { Some distress }\end{array}$ \\
\hline 17 & M. & 37 & 6 & Pf. d. d. ul... & Pf. al d. ul.... & Closure and Gas.-Ent.... & No & 6 days & 27 days & Fxcellent in every way \\
\hline 18 & M. & 29 & $\theta$ & Pf. d. d. ul.. & Pf. d. d. ul... & Closure and Gas..Ent... & Yes & 5 days & 25 days & Gained weight; good health \\
\hline 19 & F. & 41 & 5 & Acute append.... & Pf. d. d. ul...... & Perforation closed........ & Yes & 9 days & 23 days & \\
\hline 20 & M. & 22 & $\$ 1 / 2$ & Pf. d. d. ul.... & Pf. d. d. ul... & Closure and Gas.-Ent... & No & 5 days & 15 days & \\
\hline
\end{tabular}

Kenyon sucking apparatus. A soft rubber tube drainage was inserted at the lower angle of the wound. The patient made an uneventful recovery. She has not been seen or heard from since Feb. 23, 1913.

In Case 10, the perforation was situated on the anterior gastric wall about 2 inches from the pylorus. The lumen of the perforation admitted two fingers. It was the largest that I have ever seen. The duration of symptoms was fourteen hours. The patient was in shock and presented all the evidences of a man rapidly dying from a general septic peritonitis. The abdomen contained an enormous amount of thick turbid fluid, with many food particles. Numerous gas bubbles were noted. A grayish white exudate covered the entire under surface and margin of the liver. Many loops of bowel were matted together by the same sort of exudate. The perforation was closed by two rows of sutures and covered by an omental graft. The abdominal cavity was thoroughly cleansed by the sucking apparatus, and drainage instituted. It was noted that the closure embarrassed the pyloric opening very much. However, because of the precarious condition of the man, gastro-enterostomy was not attempted. Drainage was placed at the lower angle of the wound and likewise in both flanks. The patient was in very good condition for three days. He retained all liquids, both by rectum and by mouth, and we were jubilant. However, he was a man accustomed to a full diet and on the fourth day got out of bed and attempted to take food from
On opening of the abdomen in the median line, the entire anterior wall of the stomach was ecchymotic. The perforation was very small in caliber. The adjacent stomach was quite adherent to the anterior wall of the abdomen. A purse string of chromic catgut was first used to close the perforation, and then three Lembert sutures of fine linen placed to reinforce the closure. After removal of the appendix, which was found to be chronically diseased, the abdomen was closed without drainage. This man had very little reaction, and left the hospital on the fourteenth day after the operation.

In the fifteen duodenal cases multiple ulcers were noted in only one instance (Case 18). The site of the perforation in twelve of these was on the anterior wall and in the first portion of the duodenum. In two instances (Cases 15 and 17) the perforation was on the upper border and close to the pylorus. In one instance (Case 13) the perforation was on the posterior wall and about 1 inch from the pylorus. The operative procedure in dealing with these cases was as follows: In ten cases $(3,7,8,9,11,13,14,15,17$ and 18) the perforation was closed by two layers of sutures. The abdominal cavity was then thoroughly cleansed, either by the sucking apparatus or by mopping out with gauze. This was followed by a posterior no loop 
gastro-enterostomy. Recovery in each instance was most satisfactory and without any marked degree of reaction or discomfort. In five cases $(1,4,5,16$ and $20)$ the operative procedure was a simple closure of the perforation by two rows of suture and covering with an omental graft. This was always followed by a thorough cleansing of the abdominal cavity by means of the sucking apparatus or mopping out with gauze pads. In two cases ( 16 and 20) no drainage was used. In the convalescence of these cases it was noted that the patients suffered a great deal more pain and discomfort than those in which gastro-enterostomy was done.

In the operative procedure used in the series under consideration, routine doses of $1 / 4$ grain of morphin and $1 / 100$ grain of atropin were given as early as possible after deciding to operate. The anesthetic in every case was ether by the open-drop method. The site of the incision was in most cases a midright rectus one in the upper zone. The method of Gibson ${ }^{5}$ to determine gas by the use of water in the wound before opening the peritoneum was practiced in one case (Case 19) and proved very satisfactory. Gas bubbles were seen immediately after the peritoneum was opened, and before discoloration or disappearance of the water. The incision should be generous and thus permit of easy exploration and thorough cleansing of the abdominal cavity. To accomplish this cleansing, the sucking apparatus devised by Pool and Kenyon was used in the majority of cases. Its operation is simple and its results very satisfactory. Its advantage over mopping out with gauze is that there is less injury to the peritoneum, and hence lessened opportunity for the formation of adhesions.

The principal feature open to discussion in the operative procedure, when dealing with a perforation of a gastric or duodenal ulcer, is whether or not a gastroenterostomy should be added, after the closure of the perforation. There are many opinions for and against. The most recent that has come under my observation is that of Gibson, ${ }^{\circ}$ in which he says:

I reject gastro-enterostomy as a curative measure in this class of cases, notwithstanding that I have had very gratifying experience with it in the cure of chronic ulcers. I consider it unwise to do a gastro-enterostomy for a condition that is going to be cured anyhow. In a very small number of cases in which gastro-enterostomy may possibly become necessary, it can wisely be postponed until its indications are clearly recognizable. The small number of cases in which it may be necessary will probably be balanced by the number of cases in which gastro-enterostomy fails to prove satisfactory, either because it is improperly placed, too small (subsequent shrinkage), too large (a very disagreeable condition), or the rare but exceedingly grave gastrojejunal ulcer. Moreover, in dealing with acute perforations it seems wiser to do only what is absolutely necessary and not subject the patient, who may have to struggle with a possible peritonitis, to an unnecessarily long operation or risk spreading the infection by the performance of a gastroentcrostomy under imperfect aseptic conditions. The after history of this series of cases contains no instances in which we have had occasion to regret the omission of this step.

This is a clear, concise and positive statement. It has been made after a very practical and painstaking study of end-results. I regret that it was impossible for me to locate my cases in time to make Roentgenray studies. However, we have been able to interview most of them and we shall discuss the end-results at

5. Gibson: Surg., Gynec. and Obst., April, 1916.

6. Gibson: Surg., Gynec. and Obst., xxii, 393. another time. The position of Dr. Gibson deserves serious consideration and will undoubtedly furnish a safe guide to any one who considers gastroenterostomy too grave an operation to be done in the presence of a perforation. However, this attitude has not met with general practice. Eliot ${ }^{7}$ presents a series of communications from numerous advocates. Among them are Bretano and Körte of Berlin; Krogius of Helsingfors; Petrén of Lund, and W. J. Mayo, Peck and Woolsey of this country. These men do not favor it as a rule, but depend on the factors of (1) a good physical condition of the patient; (2) the narrowing of the lumen of the pylorus by the closure of the perforation, and (3) the site of the ulcer being at or near the pylorus. Deaver ${ }^{3}$ is more enthusiastic, and says that "the danger of infection of the lesser peritoneal cavity by gastro-enterostomy has been overrated." He goes further and considers the operation safe even after sixteen hours of duration of symptoms, claiming that at this time "it is simply a matter of dexterity."

In this series, gastro-enterostomy was added to the closure of the perforation in ten cases. In one it followed pylorectomy, making eleven instances. The factors that were taken into consideration as indication for the operation were: 1 . The general condition of the patient would permit it. 2. The duration of symptoms was not over ten hours. 3. The site of the perforation was close to the pylorus, and closure of it seemed to narrow the lumen to a marked degree. 4. It was assumed that by its performance the period of convalescence would be materially shortened, a factor always worthy of consideration, especially among the class of patients we meet in the wards of our city hospitals. 5. We assumed that if there were multiple ulcers. gastro-enterostomy would diminish the possibility of a second perforation. We never started out with an idea of speed; in fact, our work in each case was deliberate and careful. The average length of time taken to do the gastro-enterostomy was between thirtyfive and fifty minutes. In all cases in which this operation was done, we did not encounter any increase in infection. Neither did we have any reason to regret our procedure. The patients all made satisfactory recoveries and enjoyed their semisolid food with more relish than those on whom we did the simpler operation of suture closure. From this experience we feel that there is a place for gastro-enterostomy in the treatment of perforated ulcers of the stomach and duodenum. It is our opinion also, that in perforations at the cardiac end or on the lesser curve of the stomach, gastro-enterostomy should not be attempted. Either excision and suture closure, or simple closure should be the procedure in these cases.

In the consideration of the subject of drainage of these cases, we have come to do less of it. Especially is this true in paticnts operated on within four or five hours after perforation. Our method has been to drain the affected zone with soft rubber tubes. In only one instance have we used counter drainage. Our routine has been to drain through the original abdominal wound. At no time have we used the suprapubic drainage. By the aid of the sucking apparatus most of the abdominal fluid can easily be removed, and hence fewer and smaller drainage tubes need to be employed. However, in late cases, I would consider it unwise not to use sufficient drainage.

7. Eliot: Ann. Surg., 1v, 709. 
The postoperative care of these patients is important. The Fowler position and saline by rectum should be instituted immediately. In this series when gastroenterostomy was done, water was given by mouth in from ten to twelve hours after the operation. Four to six hours later, if the stomach remained quiet, they received milk or other fluids in increasing amounts. In the cases of simple closure of the perforation, nothing was given by mouth from thirty to thirty-six hours after operation. Then small quantities of water by mouth was allowed at frequent intervals for six to ten hours. If the stomach did not rebel, other fluids were administered in increasing amounts. All patients received an enema at the end of thirty hours and then daily for five days. At this point a cathartic, usually castor oil, was given. Soft solids were given the gastroenterostomy patients on an average of the fifth or sixth day, and increased as rapidly as possible. The simple closures did not receive this diet until on an average of the eighth or ninth day. The question of diet in the postoperative care of these patients has been the subject of considerable discussion. Gibson ${ }^{6}$ very potently says, "It is about time that we get away from the fetish of underfeeding or of the particular value of certain kinds of diet," in the after-care of these cases. I agree with this in every way. Nothing can be more of a worry to a patient than to know that he must adhere to some certain kind of rigid diet for a long time. When the patient is discharged from the hospital he should be able to handle a regular diet. However, we have cautioned against the use of very coarse food and have encleavored to educate the patient to the fact that good digestion depends mainly on mastication.

In our attempt to compile the end-results in this series, we have not had the success desired. Most of these patients belong to a migrating class and hence it is next to impossible to locate them. However, we have secured information in twelve cases of the series (Cases 1, 2, 4, 10,11, 12, 13, 14, 15, 16, 17 and 18). Of this number four (Cases 2, 10, 11 and 12) were gastric and the other eight were duodenal perforation. In the gastric cases there was one death, previously detailed. In the three remaining cases all report a gain in weight and freedom from pain. Patients 2 and 11 report that they enjoy good appetites and relish their food without any signs of stomach distress, either immediately after or later. In Case 12 , which was traumatic in origin, the patient reports that he suffers with an occasional distress and considerable gas belching beginning twenty to thirty minutes after eating and lasting about ten minutes. In the eight duodenal cases, five (Cases 13, 14, 15, 16 and 18) had gastro-enterostomy done after the closure of the perforation. All report gain in weight and freedom from any distress or pain. They seem to enjoy perfect health in every way, and assure us that they are not worried about any recurrence of their trouble. We did not receive a similar encouraging report in the three remaining cases (Cases 1, 14 and 16) in which simple closure was done. Patient 1 says that he has intermittent spells of distress coming on three to four hours after eating and is always relieved by an alkali. However, he further says that these attacks are now less frequent and of shorter duration. Patient 4 has no complaint to make except that he is "bothered with gas after eating which lasts about onehalf hour." Patient 16 was operated on, Dec. 6, 1915. We did a simple closure of the perforation, five and one-half hours after the onset of symptoms. No drainage was used. He had a small skin stitch infection and left the hospital eighteen days after the operation in apparently good condition. He was seen and interviewed, May 6, 1916, just five months after the operation. His history was that he enjoyed perfect health up to May 3, 1916. At this time he began to have rather an agonizing pain in the pit of his stomach, coming on about two hours after meals and accompanied by considerable sour regurgitation. Vomiting occurred twice. He refused Roentgen-ray examination or gastric analysis. However, by palpation and percussion there was distinct evidence of a gastric dilatation, apparently caused by some degree of pyloric stenosis. His appendix had previously been removed and we advised gastro-enterostomy. However, he elected to try his alkali once more.

\section{CONCLUSIONS}

1. The diagnosis of perforation of a gastric or duodenal ulcer should be made in the majority of cases, and the imperative indication is early operation.

2. In the treatment of cases of duodenal or prepyloric perforations, gastro-enterostomy can safely be added if patients come to operation within ten hours after the onset of symptoms.

3 . Simple closure of the perforation without gastroenterostomy is a safe routine, but later stenosis is more apt to occur.

4. Drainage can be discarded in early cases, especially if operation is performed within six hours after the onset of symptoms.

5. Early use of a liberal diet should be practiced.

6. A complete study of end-results is a necessity before any definite routine can be laid down.

208 Jefferson Avenue.

\section{ABSTRACT OF DISCUSSION}

Dr. Charies H. Peck, New York: Cases of perforation of gastric and duodenal ulcers fall into three classes: First," the acute perforation with considerable immediate leakage, the type of case which Dr. Sullivan reported; second, the chronic penetrative ulcers which are protected by inflammatory adhesions and never have any considerable leakage at one time; and third, the ulcers which leak from time to time, causing attacks of localized peritonitis, and soon becoming sealed and protected, falling again into the chronic group. A certain percentage of the cases classed as acute perforations belong in this third group. Perforations near the pylorus are apt to be accompanied by a considerable amount of inflammatory exudate and consequent narrowing of the pylorus. In this group the leakage is often moderate and the condition of the patient relatively good, hence gastro-enterostomy can of ten safely be added to the immediate closure of the perforation. The question as to whether gastro-enterostomy shall be done at the time of primary operation is an important one, and the indications laid down by Dr. Sullivan express my views very well. In the larger perforations of gastric ulcer with a good deal of immediate leakage I think it is usually unsafe to attempt to do a gastro-enterostomy, but in an ulcer near the pylorus or in duodenal ulcer with moderate leakage the operation tends to help toward the ultimate cure of the condition. I have seen closure follow without gastro-enterostomy in one case of secondary perforation of a duodenal ulcer. Dr. Gibson states that the perforation itself tends to excite a reaction in an ulcer which aids in its ultimate healing and for that reason he thinks gastro-enterostomy is never indicated. When the ulcer has perforated it is undoubtedly true that the acute reaction and inflammatory process around the ulcer tend to aid in its healing. At the Roosevelt Hospital we had between 1910 and 1915 about 30 perforated ulcers; two thirds were duodenal ulcers, one third gastric. In the duodenal cases we had no deaths from extension of the peri- 
tonitis; there were two deaths from complicating conditions. In the gastric group there were many large perforations in the center of a large indurated area, with excessive leakage and there were five deaths from extension of peritonitis which was present at the time of the original operation. In the early part of our series comparatively few gastro-enterostomies were done, but not so later. In studying the results we believe that the gastro-enterostomy did not add to the immediate mortality if the cases were properly selected. Early diagnosis and prompt operation are the keynotes of success. We are all agreed that the patients who come under observation and are treated within the first twelve or fifteen hours almost invariably recover, while in many of the later cases death occurs from extension of the peritonitis.

Dr. JohN T. Bottomley, Boston: I do not agree with Dr. Sullivan in the matter of doing away with drainage, even in early cases. I have seen the pelvis full of a nasty, turbid, dangerous fluid one and a half hours after perforation of a small gastric ulcer. With that fact in view it is in my opinion unsafe to do away with drainage in any case. The placing of a drainage tube in the pelvis greatly increases the margin of safety. We all vary in the degree of our resistance to infection and no man can measure that degree before operation, and as a matter of safety $I$ believe drainage should always be provided. I disagree also as to giving a liberal diet shortly after operation. I believe in keeping these patients on a low diet for a considerable time. They are usually not awfully sick. They recover quickly and there is no particular indication for liberal diet soon after operation.

I think a search should be made in all cases of gastric ulcer for the source of infection. We are coming to regard a large proportion of duodenal and gastric ulcers as an end result of infection in another portion of the body. This should be sought for and, if found, should be eliminated.

Dr. Harry D. Johnson, New York: Dr. Sullivan's series of cases proves two things: first, that the doctor who makes the diagnosis and refers the patient for operation is alive to the gravity of these cases, and second, that he chooses his surgeon well. If we get these cases early when we are dealing with a peritonitis due to the irritative character of the stomach or duodenal contents, all we have to do is to stop the leak and the alkaline secretions of the abdomen will neutralize the acid stomach secretion, provided there has not been the supervention of a bacterial peritonitis. At the end of twelve hours the picture changes-the chemical irritation has superimposed a bacterial invasion. The important thing is the character of the rigidity. They are so rigid that if you walk on their belly you would not sink in. The question of liver dulness as a symptom is a delay in diagnosis. That little bublble of fluid in the gastric angle is a late occurrence and should never be mentioned as a symptom of perforation of the stomach or duodenum.

Dr. Raymond Peter Sullivan, Brooklyn: The use of drainage in these cases is purely a matter of personal opinion. I cannot say just when the peritonitis will be septic. However, in dealing with early cases we assumed, from the gencral condition of the patient and the appearance of the abdominal contents, that the peritonitis present was chemical and hence limited our drainage as much as possible. The results justified our action. When these patients come to operation under ten hours after the onset of symptoms, the abdominal cavity should be cleansed thoroughly by a sucking apparatus in preference to mopping with gauze. Thus the adhesion band formation, so apt to cause secondary obstruction, will be lessened greatly. I have never used the suprapubic drainage. Our method has been to drain from the lower angle of the abdominal wound. Counter drainage was used in only one case.

It is just possible that in Boston the food may differ from what we eat in Brooklyn, and hence they see more constantly the sign of "absence of liver dulness." We have never recorded this condition. If it were present I consider it would be misleading, because so frequently a dilated colon will give modification of liver dulness. Perforation may occur at any time. If it occurs when the colon is loaded, fermentation therein may lead to modification of liver dulness.

\section{PSEUDO-APPENDICITIS *}

\author{
F. GREGORY CONNELL, M.D. \\ Fellow of the American College of Surgeons \\ osHKosH, wis.
}

In a recent clinic (June, 1915) Murphy says, "We surgeons are not talking enough about early operation in appendicitis." That, I take it, depends on the audience. At such a gathering as this, one is justified in assuming that the question of acute appendicitis is settled, at least for the time being.

The method and time of treatment, and postoperative measures are practically uniform; delay in proper treatment is usually due to uncertainty in diagnosis, and the inexcusable estimated general hospital mortality of 10 per cent. is due to failure of some one to recognize well accepted principles of surgical diagnosis or treatment.

The question of chronic appendicitis calls for attention, not because of a high mortality rate, but because of a rather disconcerting morbidity rate, a postoperative persistence of symptoms. When a patient complains of the same symptoms after appendectomy as before the operation, there is sufficient reason for belief that the original symptoms were not caused by the appendix - that the treatment was based on an incorrect diagnosis. The large and apparently increasing number of these cases calls for close attention and an analysis of all available data, in the hope of arriving at some more definite justification for making a diagnosis of "chronic appendicitis."

In gathering material for such an analysis, I have reviewed my cases of unsatisfactory results following removal of the appendix, but have excluded:

A. Acute cases.

B. Cases in which the appendix was removed during the course of an operation for other conditions.

C. Cases in which ordinary sequelae of laparotomy have occurred, such as. hernia, ventral or inguinal, ileus, fistula, phlebitis, etc.

After excluding the foregoing those remaining are unquestionably due to mistaken diagnosis and may be divided into:

D. Cases in which the true pathologic lesion, and proper diagnosis, has been demonstrated at a subsequent operation, or is reasonably evident, such as gastric and duodenal ulcer, cholecystitis, pancreatitis, lesions of the genito-urinary tract, and of the uterus and adnexa, tuberculosis, syphilis, malignancy, orthopedic conditions, and spinal cord lesions.

E. Cases to which I shall direct attention, namely, those in which the persistence of the symptoms has not been rationally explained, and which might well be termed pseudo-appendicitis.

After excluding Classes A, B, C and D, I found between January, 1909, and January, 1916, records of eighty-seven cases in which the removal of the appendix, for chronic appendicitis, or interval operation for appendicitis, had not been followed by relief of symptoms; forty-eight of the eighty-seven patients were operated on by myself; 212 patients were operated on during this interval with such a diagnosis.

Forty-eight in 212 cases seems a high percentage of unsatisfactory results, and it is because of this fact, coupled with the paucity of similar reports on remote results in the literature, that the present analysis has been made.

* Read before the Section on Surgery, General and Abdominal, a the Sixty-Seventh Annua! Session of the American Medical Association, Detroit, June, 1916. 